\title{
SEMICLASSICAL RESOLVENT ESTIMATES AT TRAPPED SETS
}

\author{
KIRIL DATCHEV AND ANDRÁS VASY
}

\begin{abstract}
We extend our recent results on propagation of semiclassical resolvent estimates through trapped sets when a priori polynomial resolvent bounds hold. Previously we obtained non-trapping estimates in trapping situations when the resolvent was sandwiched between cutoffs $\chi$ microlocally supported away from the trapping: $\left\|\chi R_{h}(E+i 0) \chi\right\|=\mathcal{O}\left(h^{-1}\right)$, a microlocal version of a result of Burq and Cardoso-Vodev. We now allow one of the two cutoffs, $\tilde{\chi}$, to be supported at the trapped set, giving $\left\|\chi R_{h}(E+i 0) \tilde{\chi}\right\|=\mathcal{O}\left(\sqrt{a(h)} h^{-1}\right)$ when the a priori bound is $\left\|\tilde{\chi} R_{h}(E+i 0) \tilde{\chi}\right\|=\mathcal{O}\left(a(h) h^{-1}\right)$.
\end{abstract}

In this brief article we extend the resolvent and propagation estimates of [DaVa10].

Let $(X, g)$ be a Riemannian manifold which is asymptotically conic or asymptotically hyperbolic in the sense of [DaVa10], let $V \in C_{0}^{\infty}(X)$ be real valued, let $P=h^{2} \Delta_{g}+V(x)$, where $\Delta_{g} \geq 0$, and fix $E>0$.

Theorem 1. DaVa10, Theorem 1.2] Suppose that for any $\chi_{0} \in C_{0}^{\infty}(X)$ there exist $C_{0}, k, h_{0}>$ 0 such that for any $\varepsilon>0, h \in\left(0, h_{0}\right]$ we have

$$
\left\|\chi_{0}\left(h^{2} \Delta_{g}+V-E-i \varepsilon\right)^{-1} \chi_{0}\right\|_{L^{2}(X) \rightarrow L^{2}(X)} \leq C_{0} h^{-k} .
$$

Let $K_{E} \subset T^{*} X$ be the set of trapped bicharacteristics at energy $E$, and suppose that $b \in$ $C_{0}^{\infty}\left(T^{*} X\right)$ is identically 1 near $K_{E}$. Then there exist $C_{1}, h_{1}>0$ such that for any $\varepsilon>0$, $h \in\left(0, h_{1}\right]$ we have the following nontrapping estimate:

$$
\left\|\langle r\rangle^{-1 / 2-\delta}(1-\mathrm{Op}(b))\left(h^{2} \Delta_{g}+V-E-i \varepsilon\right)^{-1}(1-\mathrm{Op}(b))\langle r\rangle^{-1 / 2-\delta}\right\|_{L^{2}(X) \rightarrow L^{2}(X)} \leq C_{1} h^{-1} .
$$

Here by bicharacteristics at energy $E$ we mean integral curves in $p^{-1}(E)$ of the Hamiltonian vector field $H_{p}$ of the Hamiltonian $p=|\xi|^{2}+V(x)$, and the trapped ones are those which remain in a compact set for all time. We use the notation $r=r(z)=d_{g}\left(z, z_{0}\right)$, where $d_{g}$ is the distance function on $X$ induced by $g$ and $z_{0} \in X$ is fixed but arbitrary.

If $K_{E}=\varnothing$ then (1) holds with $k=1$. If $K_{E} \neq \varnothing$ but the trapping is sufficiently 'mild', then (1) holds for some $k>1$ : see DaVa10] for details and examples. The point is that the losses in (11) due to trapping are removed when the resolvent is cutoff away from $K_{E}$. Theorem 1 is a more precise and microlocal version of an earlier result of Burq [Bur02] and Cardoso and

Date: May 29, 2012.

2010 Mathematics Subject Classification. 58J47, 35L05.

Key words and phrases. Resolvent estimates, trapping, propagation of singularities.

The first author is partially supported by a National Science Foundation postdoctoral fellowship, and the second author is partially supported by the National Science Foundation under grants DMS-0801226 and DMS-1068742. 
Vodev [CaVo02, but the assumption (1) is not needed in [Bur02, CaVo02. See [DaVa10] for additional background and references for semiclassical resolvent estimates and trapping.

In this paper we prove that an improvement over the a priori estimate (11) holds even when one of the factors of $(1-\mathrm{Op}(b))$ is removed:

Theorem 2. Suppose that there exist $k>0$ and $a(h) \leq h^{-k}$ such that for any $\chi_{0} \in C_{0}^{\infty}(X)$ there exists $h_{0}>0$ such that for any $\varepsilon>0, h \in\left(0, h_{0}\right]$ we have

$$
\left\|\chi_{0}\left(h^{2} \Delta_{g}+V-E-i \varepsilon\right)^{-1} \chi_{0}\right\|_{L^{2}(X) \rightarrow L^{2}(X)} \leq a(h) / h .
$$

Suppose that $b \in C_{0}^{\infty}\left(T^{*} X\right)$ is identically 1 near $K_{E}$. Then there exist $C_{1}, h_{1}>0$ such that for any $\varepsilon>0, h \in\left(0, h_{1}\right]$,

$$
\left\|\langle r\rangle^{-1 / 2-\delta}(1-\mathrm{Op}(b))\left(h^{2} \Delta_{g}+V-E-i \varepsilon\right)^{-1}\langle r\rangle^{-1 / 2-\delta}\right\|_{L^{2}(X) \rightarrow L^{2}(X)} \leq C_{1} \sqrt{a(h)} / h .
$$

Note that by taking adjoints, analogous estimates follow if $1-\mathrm{Op}(b)$ is placed to the other side of $\left(h^{2} \Delta_{g}+V-E-i \varepsilon\right)^{-1}$.

Such results were proved by Burq and Zworski [BuZw04, Theorem A] and Christianson Chr07, (1.6)] when $K_{E}$ consists of a single hyperbolic orbit. Theorem 2 implies an optimal semiclassical resolvent estimate for the example operator of [DaVa10, §5.3]: it improves [DaVa10, (5.5)] to

$$
\left\|\chi_{0}(P-\lambda)^{-1} \chi_{0}\right\| \leq C \log (1 / h) / h .
$$

Further, this improved estimate can be used to extend polynomial resolvent estimates from complex absorbing potentials to analogous estimates for damped wave equations; this is a result of Christianson, Schenk, Wunsch and the second author [CSVW].

Theorems 1 and 2 follow from microlocal propagation estimates in a neighborhood of $K_{E}$, or more generally in a neighborhood of a suitable compact invariant subset of a bicharacteristic flow.

To state the general results, suppose $X$ is a manifold, $P \in \Psi^{m, 0}(X)$ a self adjoint, order $m>0$, semiclassical pseudodifferential operator on $X$, with principal symbol $p$. For $I \subset \mathbb{R}$ compact and fixed, denote the characteristic set by $\Sigma=p^{-1}(I)$, and suppose that the projection to the base, $\pi: \Sigma \rightarrow X$, is proper (it is sufficient, for example, to have $p$ classically elliptic). Suppose that $\Gamma \Subset T^{*} X$ is invariant under the bicharacteristic flow in $\Sigma$. Define the forward, resp. backward flowout $\Gamma_{+}$, resp. $\Gamma_{-}$, of $\Gamma$ as the set of points $\rho \in \Sigma$, from which the backward, resp. forward bicharacteristic segments tend to $\Gamma$, i.e. for any neighborhood $O$ of $\Gamma$ there exists $T>0$ such that $-t \geq T$, resp. $t \geq T$, implies $\gamma(t) \in O$, where $\gamma$ is the bicharacteristic with $\gamma(0)=\rho$. Here we think of $\Gamma$ as the trapped set or as part of the trapped set, hence points in $\Gamma_{-}$, resp. $\Gamma_{+}$are backward, resp. forward, trapped. Suppose $V$, $W$ are neighborhoods of $\Gamma$ with $\bar{V} \subset W, \bar{W}$ compact. Suppose also that

$$
\text { If } \rho \in W \backslash \Gamma_{+} \text {, resp. } \rho \in W \backslash \Gamma_{-},
$$

then the backward, resp. forward bicharacteristic from $\rho$ intersects $W \backslash \bar{V}$.

This means that all bicharacterstics in $V$ which stay in $V$ for all time tend to $\Gamma$. 
The main result of [DaVa10], from which the other results in the paper follow, is the following:

Theorem 3. [DaVa10, Theorem 1.3] Suppose that $\|u\|_{H_{h}^{-N}} \leq h^{-N}$ for some $N \in \mathbb{N}$ and $(P-\lambda) u=f, \operatorname{Re} \lambda \in I$ and $\operatorname{Im} \lambda \geq-\mathcal{O}\left(h^{\infty}\right)$. Suppose $f$ is $\mathcal{O}(1)$ on $W, \mathrm{WF}_{h}(f) \cap \bar{V}=\emptyset$, and $u$ is $\mathcal{O}\left(h^{-1}\right)$ on $W \cap \Gamma_{-} \backslash \bar{V}$. Then $u$ is $\mathcal{O}\left(h^{-1}\right)$ on $W \cap \Gamma_{+} \backslash \Gamma$.

Here we say that $u$ is $\mathcal{O}(a(h))$ at $\rho \in T^{*} X$ if there exists $B \in \Psi^{0,0}(X)$ elliptic at $\rho$ with $\|B u\|_{L^{2}}=\mathcal{O}(a(h))$. We say $u$ is $\mathcal{O}(a(h))$ on a set $E \subset T^{*} X$ if it is $\left.\mathcal{O}(a(h))\right)$ at each $\rho \in E$.

Note that there is no conclusion on $u$ at $\Gamma$; typically it will be merely $\mathcal{O}\left(h^{-N}\right)$ there. However, to obtain $\mathcal{O}\left(h^{-1}\right)$ bounds for $u$ on $\Gamma_{+}$we only needed to assume $\mathcal{O}\left(h^{-1}\right)$ bounds for $u$ on $\Gamma_{-}$and nowhere else. Note also that by the propagation of singularities, if $u$ is $\mathcal{O}\left(h^{-1}\right)$ at one point on any bicharacteristic, then it is such on the whole forward bicharacteristic. If $|\operatorname{Im} \lambda|=\mathcal{O}\left(h^{\infty}\right)$ then the same is true for backward bicharacteristics.

In this paper we show that a (lesser) improvement on the a priori bound holds even when $f$ is not assumed to vanish microlocally near $\Gamma$ :

Theorem 4. Suppose that $\|u\|_{H_{h}^{-N}} \leq h^{-N}$ for some $N \in \mathbb{N}$ and $(P-\lambda) u=f, \operatorname{Re} \lambda \in I$ and $\operatorname{Im} \lambda \geq-\mathcal{O}\left(h^{\infty}\right)$. Suppose $f$ is $\mathcal{O}(1)$ on $W$, u is $\mathcal{O}\left(a(h) h^{-1}\right)$ on $W$, and $u$ is $\mathcal{O}\left(h^{-1}\right)$ on $W \cap \Gamma_{-} \backslash \bar{V}$. Then $u$ is $\mathcal{O}\left(\sqrt{a(h)} h^{-1}\right)$ on $W \cap \Gamma_{+} \backslash \Gamma$.

In [DaVa10] Theorem 1 is deduced from Theorem 3. Theorem 2 follows from Theorem 4 by the same argument.

Proof of Theorem 4. The argument is a simple modification of the argument of [DaVa10, End of Section 4, Proof of Theorem 1.3]; we follow the notation of this proof. Recall first from [DaVa10, Lemma 4.1] that if $U_{-}$is a neighborhood of $\left(\Gamma_{-} \backslash \Gamma\right) \cap(\bar{W} \backslash V)$ then there is a neighborhood $U \subset V$ of $\Gamma$ such that if $\alpha \in U \backslash \Gamma_{+}$then the backward bicharacteristic from $\alpha$ enters $U_{-}$. Thus, if one assumes that $u$ is $\mathcal{O}\left(h^{-1}\right)$ on $\Gamma_{-}$and $f$ is $\mathcal{O}(1)$ on $\bar{V}$, it follows that that $u$ is $\mathcal{O}\left(h^{-1}\right)$ on $U \backslash \Gamma_{+}$, provided $U_{-}$is chosen small enough that $u$ is $\mathcal{O}\left(h^{-1}\right)$ on $U_{-}$. Note also that, because $U \subset V, f$ is $\mathcal{O}(1)$ on $U$. We will show that $u$ is $\mathcal{O}\left(\sqrt{a(h)} h^{-1}\right)$ on $U \cap \Gamma_{+} \backslash \Gamma$ : the conclusion on the larger set $W \cap \Gamma_{+} \backslash \Gamma$ follows by propagation of singularities.

Next, [DaVa10, Lemma 4.3] states that if $U_{1}$ and $U_{0}$ are open sets with $\Gamma \subset U_{1} \Subset U_{0} \Subset U$ then there exists a nonnegative function $q \in C_{0}^{\infty}(U)$ such that

$$
q=1 \text { near } \Gamma, \quad H_{p} q \leq 0 \text { near } \Gamma_{+}, \quad H_{p} q<0 \text { on } \Gamma_{+}^{\overline{U_{0}}} \backslash U_{1} .
$$

Moreover, we can take $q$ such that both $\sqrt{q}$ and $\sqrt{-H_{p} q}$ are smooth near $\Gamma_{+}$.

Remark. The last paragraph in the proof of [DaVa10, Lemma 4.3] should be replaced by the following: To make $\sqrt{-H_{p} \tilde{q}}$ smooth, let $\psi(s)=0$ for $s \leq 0, \psi(s)=e^{-1 / s}$ for $s>0$, and assume as we may that $U_{\rho} \cap \mathcal{S}_{\rho}$ is a ball with respect to a Euclidean metric (in local coordinates near $\rho$ ) of radius $r_{\rho}>0$ around $\rho$. We then choose $\varphi_{\rho}$ to behave like $\psi\left(r_{\rho}^{\prime 2}-|.|^{2}\right)$ with $r_{\rho}^{\prime}<r_{\rho}$ for $|$.$| close to r_{\rho}^{\prime}$, bounded away from 0 for smaller values of $|$.$| , and choose -\chi_{\rho}^{\prime}$ 
to vanish like $\psi$ at the boundary of its support. That sums of products of such functions have smooth square roots follows from [Hö94, Lemma 24.4.8].

The proof of Theorem 4 proceeds by induction: we show that if $u$ is $\mathcal{O}\left(h^{k}\right)$ on a sufficiently large compact subset of $U \cap \Gamma_{+} \backslash \Gamma$, then $u$ is $\mathcal{O}\left(h^{k+1 / 2}\right)$ on $\Gamma_{+}^{\bar{U}_{0}} \backslash U_{1}$, provided $\sqrt{a(h)} h^{-1} \leq$ $C h^{k+1 / 2}$.

Now let $U_{-}$be an open neighborhood of $\Gamma_{+} \cap \operatorname{supp} q$ which is sufficiently small that $H_{p} q \leq 0$ on $U_{-}$and that $\sqrt{-H_{p} q}$ is smooth on $U_{-}$. Let $U_{+}$be an open neighborhood of $\operatorname{supp} q \backslash U_{-}$ whose closure is disjoint from $\Gamma_{+}$and from $T^{*} X \backslash \bar{U}$. Define $\phi_{ \pm} \in C^{\infty}\left(U_{+} \cup U_{-}\right)$with $\operatorname{supp} \phi_{ \pm} \subset U_{ \pm}$and with $\phi_{+}^{2}+\phi_{-}^{2}=1$ near $\operatorname{supp} q$.

Put

$$
b \stackrel{\text { def }}{=} \phi_{-} \sqrt{-H_{p} q^{2}}, \quad e \stackrel{\text { def }}{=} \phi_{+}^{2} H_{p} q^{2} .
$$

Let $Q, B, E \in \Psi^{-\infty, 0}(X)$ have principal symbols $q, b, e$, and microsupports $\operatorname{supp} q, \operatorname{supp} b$, $\operatorname{supp} e$, so that

$$
\frac{i}{h}\left[P, Q^{*} Q\right]=-B^{*} B+E+h F,
$$

with $F \in \Psi^{-\infty, 0}(X)$ such that $\mathrm{WF}_{h}^{\prime} F \subset \operatorname{supp} d q \subset U \backslash \Gamma$. But

$$
\begin{aligned}
& \frac{i}{h}\left\langle\left[P, Q^{*} Q\right] u, u\right\rangle=\frac{2}{h} \operatorname{Im}\left\langle Q^{*} Q(P-\lambda) u, u\right\rangle+\frac{2}{h}\left\langle Q^{*} Q \operatorname{Im} \lambda u, u\right\rangle \\
& \geq-2 h^{-1}\|Q(P-\lambda) u\|\|Q u\|-\mathcal{O}\left(h^{\infty}\right)\|u\|^{2} \geq-C h^{-2} a(h)-\mathcal{O}\left(h^{\infty}\right),
\end{aligned}
$$

where we used $\operatorname{Im} \lambda \geq-\mathcal{O}\left(h^{\infty}\right)$ and that on $\operatorname{supp} q,(P-\lambda) u$ is $\mathcal{O}(1)$. So

$$
\|B u\|^{2} \leq\langle E u, u\rangle+h\langle F u, u\rangle+C h^{-2} a(h)+\mathcal{O}\left(h^{\infty}\right) .
$$

But $|\langle E u, u\rangle| \leq C h^{-2}$ because $\mathrm{WF}_{h}^{\prime} E \cap \Gamma_{+}=\emptyset$ gives that $u$ is $\mathcal{O}\left(h^{-1}\right)$ on $\mathrm{WF}_{h}^{\prime} E$ by the first paragraph of the proof. Meanwhile $|\langle F u, u\rangle| \leq C\left(h^{-2}+h^{2 k}\right)$ because all points of $\mathrm{WF}_{h}^{\prime} F$ are either in $U \backslash \Gamma_{+}$, where we know $u$ is $\mathcal{O}\left(h^{-1}\right)$ from the first paragraph of the proof, or on a single compact subset of $U \cap \Gamma_{+} \backslash \Gamma$, where we know that $u$ is $\mathcal{O}\left(h^{k}\right)$ by inductive hypothesis.

Since $b=\sqrt{-H_{p} q^{2}}>0$ on $\Gamma_{+}^{\overline{U_{0}}} \backslash U_{1}$, we can use microlocal elliptic regularity to conclude that $u$ is $\mathcal{O}\left(h^{k+1 / 2}\right)$ on $\Gamma_{+}^{\bar{U}_{0}} \backslash U_{1}$, as desired.

\section{REFERENCES}

[Bur02] Nicolas Burq. Lower bounds for shape resonances widths of long range Schrödinger operators. Amer. J. Math., 124(4):677-735, 2002.

[BuZw04] Nicolas Burq and Maciej Zworski. Geometric control in the presence of a black box. J. Amer. Math. Soc. 17:2, 443-471, 2004.

[CaVo02] Fernando Cardoso and Georgi Vodev. Uniform estimates of the resolvent of the Laplace-Beltrami operator on infinite volume Riemannian manifolds. II. Ann. Henri Poincaré, 3(4):673-691, 2002.

[Chr07] Hans Christianson. Semiclassical non-concentration near hyperbolic orbits. J. Funct. Anal. 246(2):145-195, 2007. Corrigendum. J. Funct. Anal. 258(3):1060-1065, 2010.

[CSVW] Hans Christianson, Emmanuel Schenk, András Vasy and Jared Wunsch. From resolvent estimates to damped waves. In preparation.

[DaVa10] Kiril Datchev and András Vasy. Propagation through trapped sets and semiclassical resolvent estimates. Annales de l'Institut Fourier, to appear. 
[Hö94] Lars Hörmander, The Analysis of Linear Partial Differential Operators. III. Pseudo-Differential Operators, Springer Verlag, 1994.

Department of Mathematics, Massachusetts Institute of Technology, Cambridge, MA 021394397, U.S.A.

E-mail address: datchev@math.mit.edu

Department of Mathematics, Stanford University, Stanford, CA 94305-2125, U.S.A.

E-mail address: andras@math.stanford.edu 